\title{
DESIGN AND IMPLEMENT AN IOT CLOUD FOR FIELD SURVEY BASED ON COAP PROTOCOL
}

\author{
${ }^{*}$ Hadeel H. Azeez'
}

\section{Mahmood Z. Abdullah'1}

1) Computer engineering department, College of Engineering, Mustansiriyah University, Baghdad, Iraq

\begin{abstract}
Urban planning for smart cities requires collecting big real-time data, specially geolocation data from GPS sensors to use in many services like finding the best location for new schools so this data must be stored in a secure place with low cost and because the storage services offered from different cloud providers like Google, Amazon Web Service, Azure, etc., is not free. For these reasons, this study proposed Internet of Things (loT) cloud architecture using Raspberry Pi model B+ as a cloud server with MySQL database services to provide free and secure storage at a low cost. The main contributions of this study lie in the Constrained Application Protocol (CoAP) server hosted in raspberry $\mathrm{Pi}$ to offer services in the proposed architecture of the IoT cloud with different scenarios to know the proposed architecture's ability for handling many user requests per second in terms of standard division, average elapsed time, error rate, throughput, and a number of real stored data in the database. AS a result, the proposed architecture handled 150 requests per second in realtime with an elapsed time of 1186 milliseconds without any error or data loss.
\end{abstract}

Keywords: Raspberry Pi, CoAP protocol, IoT, Cloud.

\section{Introduction}

The Internet of Things (IoT) is a technology that links any objects/things using the internet to communicate with one another and deliver better services to consumers making life simpler for them. Sensors, actuators, smartphones, and other intelligent devices are used as things. Smart homes, smart cities, and smart agriculture are just a few examples of IoT applications. Kevin Ashton initially proposed the Internet of Things concept in 1999 while working with Auto-ID Center to establish a network of items utilizing Radio-frequency Identification (RFID)[1][2]. According to the official NIST definition, Cloud Computing is "a model for enabling ubiquitous, convenient, on-demand network access to a shared pool of configurable computing resources (e.g., networks, servers, storage, applications, and services) that can be rapidly provisioned and released with minimal management effort or service provider interaction"[3]. Because IoT has limited processing power and storage, it raises challenges such as performance, security, reliability, and privacy. The use of IoT in conjunction with the cloud is more advantageous in gaining access to limitless capabilities such as storage and processing capacity. IoT can even

help the cloud by allowing it to expand its capabilities by allowing it to interact with realworld objects in a more dynamic and dispersed manner and real-time delivery of a large variety

*Corresponding Author: egma023@uomustansiriyah.edu.iq 
of services [4]. The Cloud is useful in IoT situations for various reasons, Cloud connections have become an essential aspect of IoT implementations. The key benefit of connecting IoT devices to the Cloud is remote access to the data they gather (for example, a temperature sensor) and their actions (like turn on a light). Other features include device administration as well as limitless data storage. Data visualization and analytics are improved with the use of a centralized dashboard [5]. We can enhance the usage of existing technology supplied in cloud settings by combining IoT and Cloud. Cloud storage is utilized to store applications and data that employ Internet of Things technologies with this integration. Fig. 1 depicts the combination of IoT and Cloud technologies. The cloud provides wireless users with access to all of the information and applications required for IoT connection. [6].

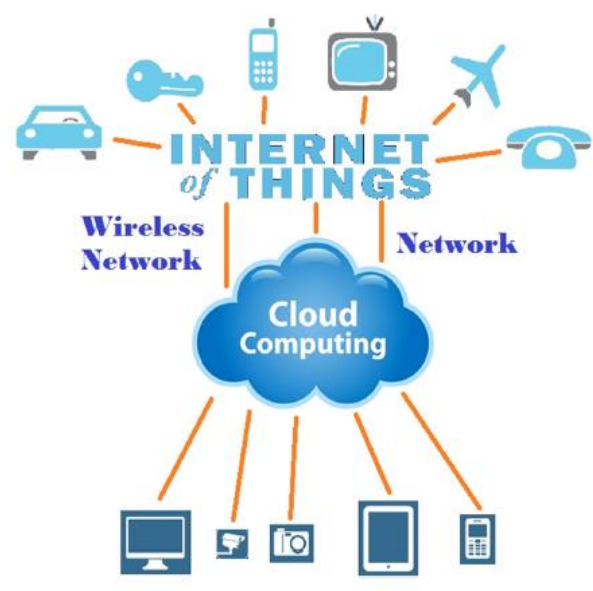

Figure 1.Cloud for IoT [6]

\section{Literature Review}

$\mathrm{Lu} \mathrm{Hou}$ et al. provided information on the IoT cloud's design, implementation, and performance. Investigated several possible IoT cloud application scenarios and discussed architecture in terms of each component's capability. In addition, the IoT cloud's implementation specifics, as well as the services it provides, are given. The key contributions were in the use of a mixture of HTTP and Message Queuing Telemetry Transport (MQTT) servers to provide IoT services in the IoT cloud architecture, as well as other ways to ensure high performance [7]. Thomas Lee Scott and Amna Eleyan investigated using the CoAP to send sensor data to the cloud. They were designed to see how CoAP fits into the IoT ecosystem and what benefits it has over other IoT protocols. Using a Raspberry Pi and sensors as IoT endpoints, a CoAP-based IoT architecture was presented [8]. Dejana Ugrenovic and Gordana Gardasevic designed IoT remote healthcare monitoring system which gives information about the patient's status via a Web browser. They employed Contiki OS with the 6LoWPAN protocol stack and Cooja, the built-in Contiki emulator, for simulation[9]. Mohammad Aizuddin Daud et al. created various testbeds based on performance factors to fulfill the device's resource requirements. The study's findings and analysis support the performance conclusion that CoAP is quicker than HTTP with lesser data. The study strengthened the use of CoAP for constrained devices concerning the limited resources, so adding to how data is managed in any IoT ecosystem [10].

As mention above, these studies are either use a public cloud or virtual machine server for storing data or test the performance based on IoT protocol. Our design proposed Raspberry Pi as a cloud with MySQL service.CoAP protocol has been used as an IoT protocol.

\section{Constrained Application Protocol (CoAP):}

For IoT applications, the IETF suggested this protocol to fit communication among resourceconstrained and unsynchronized devices, providing flow management, dependable delivery, and easy congestion control. Because of its simplicity, short message size, and 
minimal code footprint, CoAP uses User Datagram Protocol (UDP) to manage resources, decrease bandwidth needs, and so on, and before the start of transmission, eliminate the expense of TCP handshake overhead [11]. The CoAP has a client/server architecture similar to HTTP. A client requests a server that includes a method code, such as GET, PUT, POST, or DELETE. The server answers with a payload and a response code after receiving a request [12] There are four sorts of messages in the CoAP protocol as shown in Fig.2:confirmable,non-confirmable, acknowledgment, and reset [13].

- Confirmable (CON) messages need an acknowledgment (ACK), and the answer can be provided in the same ACK message (synchronously) or a separate message if extra calculation time is required (asynchronously).

- Non-Confirmable (NON): a NON mean message does not need an ACK.

- Acknowledgment (ACK): sent in response to CON messages, to confirm their reception.

- Reset (RST): sent as a response of a message that could not be processed[14].

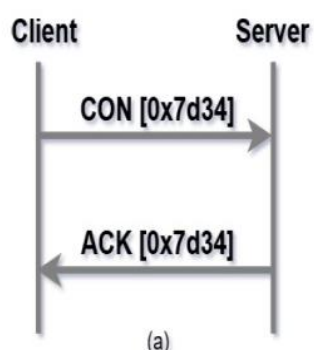

Figure 2. Messages types exchanged in CoAP: (a) Confirmable Messages; (b) Non-Confirmable Messages [14]

\section{Methodology}

The proposed architecture has consisted of two layers the client layer and the cloud layer. As shown in Fig. 3. The two layers are explained below:

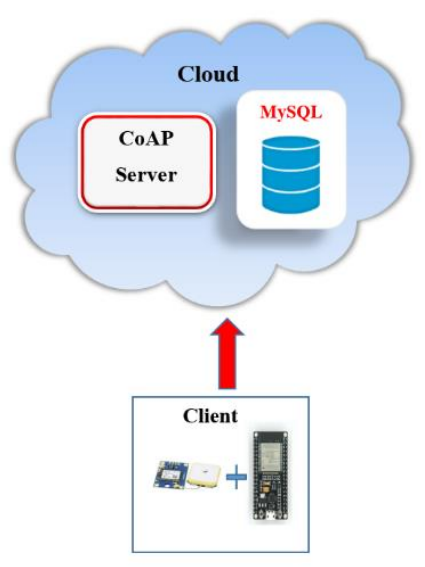

Figure 3. The Proposed IoT Cloud

\subsection{Client Layer}

This layer represents the client send GPS locations to the cloud using CoAP protocols to store them in the database. This layer consists of a GPS NEO-6M module connected to ESP WROOM-32 programmed using a C programming language. The GPS is connected to the ESP through jumper wires on the breadboard. On the other terminal, ESP is joined by an Android cable to get the required operating power source, as shown in Fig. 4. To measure the ability of the proposed architectures tens of clients are required. Due to the high cost of using tens of GPS and ESP, the solution offered to simulate virtual tens of clients to generate a load. Apache JMeter software is used.

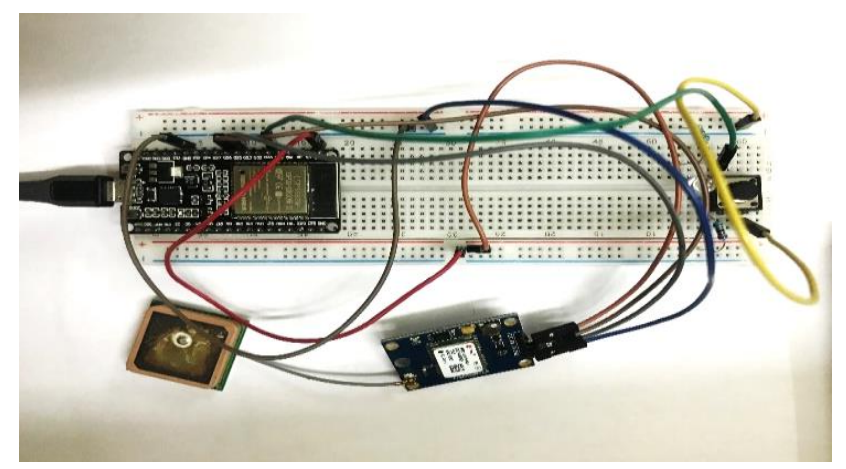

Figure 4.Show GPS and ESP WROOM-32 connection 


\section{Apache JMeter}

It is an open-source tool that is used to test functional behavior and measure some aspects of the performance metrics. JMeter tool is installed on a Laptop with windows 10 (ThinkPad core i7 CPU), 8 GByte of Dynamic Random Access Memory (DRAM), 250 GByte of permanent storage. Apache JMeter allows performing load tests on various protocols and technologies by defining a test plan. Thread groups are added from the test plan to set the required number of threads/users, time, and loop iteration. All controllers and samplers for a test must reside under a thread group. Samplers for numerous protocols, including HTTP, MQTT, and others, are included in JMeter. JMeter may be extended to provide samplers for protocols that aren't included in the upstream version. For example, CoAP Sampler was not included in the Apache JMeter plugin, so it has been added to it to be used for load testing. Listeners are used for gathering the results of a running test to be further analyzed. In addition, listeners provide the ability to save the data to a file for later use [15] [16]. Fig.5. shows the thread group of Apache JMeter.

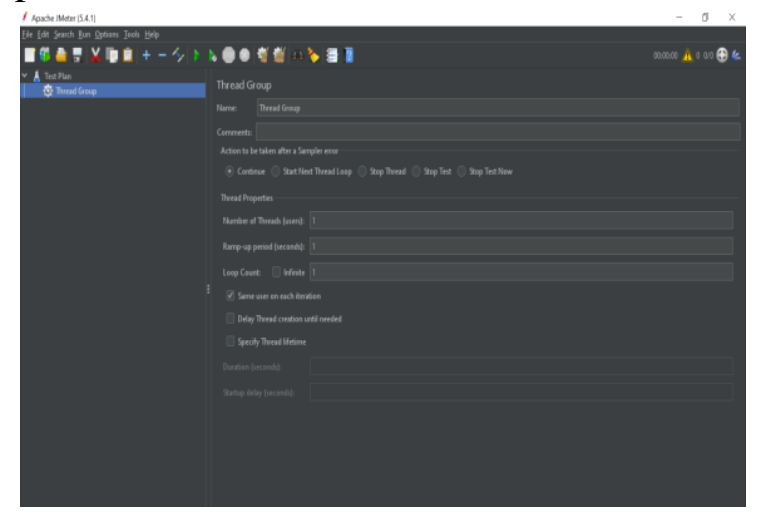

Figure 5.The thread group of Apache JMeter

\subsection{Cloud layer}

A Raspberry Pi 3 Model B+ was utilized as the cloud, which simulates a cloud server with a MySQL database for storing data from the client. The Raspberry $\mathrm{Pi}$ is a credit-card-sized computer-based development board running on either a power supply or battery power. It may be used and connected much like any other computer, using a mouse, keyboard, and screen to accomplish computing tasks. The Raspberry Pi 3 Model $\mathrm{B}+$ board has a $1.4 \mathrm{GHz}$ quad-core 64-bit CPU, dual-band wireless LAN, and a microSD card slot, Bluetooth 4.2/BLE, four USB ports, a MicroSD card slot, memory, video/audio outputs, HDMI, Ethernet, audio, and a CSI camera connection are among the features. [17] [18]. Fig.6 shows the Raspberry Pi model $\mathrm{B}+$.

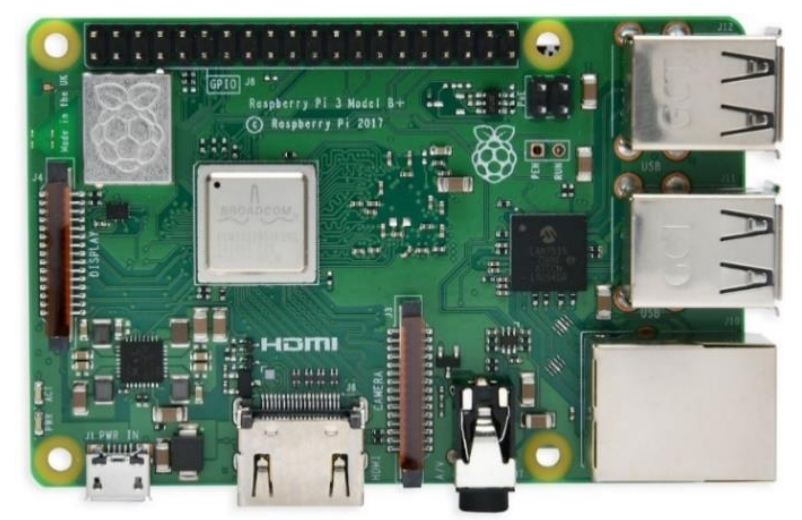

Figure 6. Raspberry Pi 3 Model B+[17]

SSH login on a remote control computer linked to the Raspberry $\mathrm{Pi}$ on the network allows access to the Raspberry Pi shell. A virtual network computing server (VNC) is also installed on Raspberry $\mathrm{Pi}$, which uses to display the Raspberry Pi's GUI-enabled desktop. SSH login with PuTTY gives us fantastic access to the command line text environment.

The following are the cloud design steps.

1. The Raspberry Pi was loaded with the Raspbian Linux distribution based on Debian, optimized for the Raspberry Pi.

2. Install PHP and PHPMyAdmin. 
3. Install the MySQL database and create a database with several columns to store the locations, as shown in Fig.7.

4. Install Python to implement CoAPthon.

5. Install CoAPthon, an open-source Python library that acts as a CoAP server that implements the CoAP protocol and its primary extensions, including blockwise transmission, resource observing, and the CoRE link format [19].

6. Modified the server code of CoAPthon to save the received data on a MySQL database.

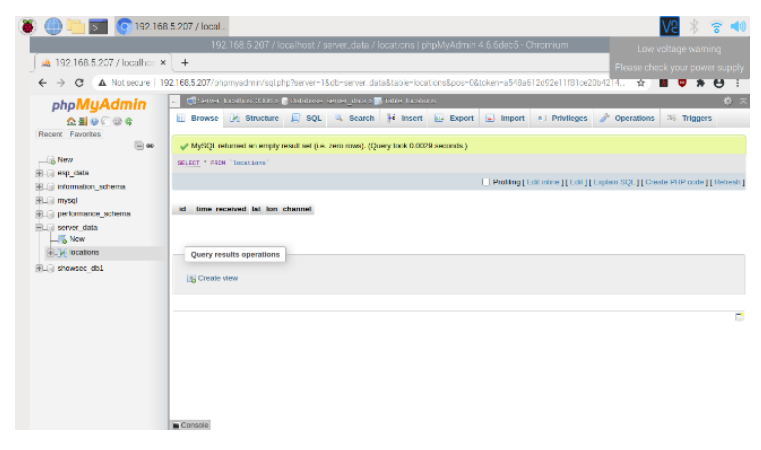

Figure 7. MySQL Database

\section{Test and Results}

For the test, we simulate 10 to 180 virtual clients (threads) in seven scenarios $(10,30,60$, $90,120,150$, and 180) using JMeter with the CoAP sampler as a load generator. Virtual clients send requests with GPS location (longitude and latitude) parameter to URL, representing the IP address of Raspberry Pi and CoAP sever run on it. Then, this location stored in the MySQL hosted on it. For this experimentation, only the CoAP POST request with CON message type has been taken into consideration. These techniques caused the server to deliver acknowledgement responses in accordance with the client's request. By the end of each test, JMeter will provide the performance measurements, such as standard division, average elapsed time, error rate, and throughput. The throughput is calculated as the number of successful requests handled by the targeted server per unit of time. The time is calculated from the start of the first sample sends to the end of the last sample. JMeter measures the elapsed time in milliseconds for requests that were sent and received a response back successfully from the server. Fig. 8 illustrates a sequence diagram of one request of the test.

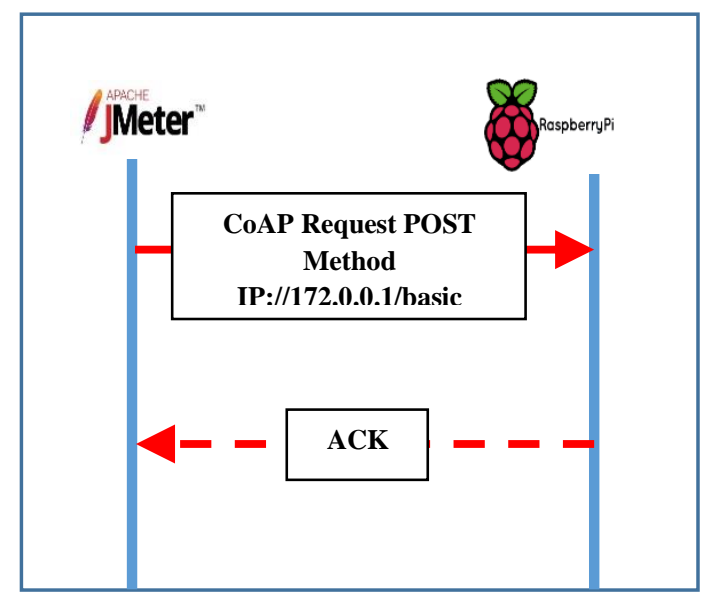

Figure 8. Sequence diagram of one CoAP request and response back from the server

Table 1 shows (standard division, average elapsed time, error rate, and throughput) results, and the number of real stored data in each case after executing each scenario. Fig.9 shows the average elapsed time increase with the number of the clients increase. This could be related to the CoAPthon implementation according to [19]. Also, the time-out error occurred which represents the maximum time that a client is prepared to wait for a response (ACK) back from a server [20]. Fig. 10 shows the data stored in MySQL. 
Table 1.The result for all cases

\begin{tabular}{|c|c|c|c|c|c|}
\hline 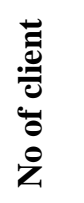 & 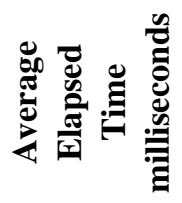 & $\begin{array}{l}\dot{\vec{U}} \\
\dot{0} \\
\dot{\vec{D}}\end{array}$ & $\begin{array}{l}0 \\
\dot{0} \\
\vdots\end{array}$ & 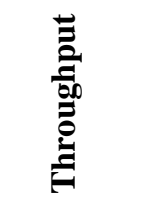 & 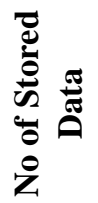 \\
\hline 10 & 42 & 36.66 & $0.00 \%$ & 9.58773 & 10 \\
\hline 30 & 51 & 27.47 & $0.00 \%$ & 30.21148 & 30 \\
\hline 60 & 689 & 1261.7 & $10.00 \%$ & 11.21076 & 60 \\
\hline 90 & 664 & 1007.07 & $5.56 \%$ & 15.97161 & 90 \\
\hline 120 & 1273 & 1259.02 & $5.83 \%$ & 17.95601 & 120 \\
\hline 150 & 1186 & 691.69 & $0.00 \%$ & 44.027 & 150 \\
\hline 180 & 2788 & 3136.8 & $20.00 \%$ & 18.27782 & 154 \\
\hline
\end{tabular}

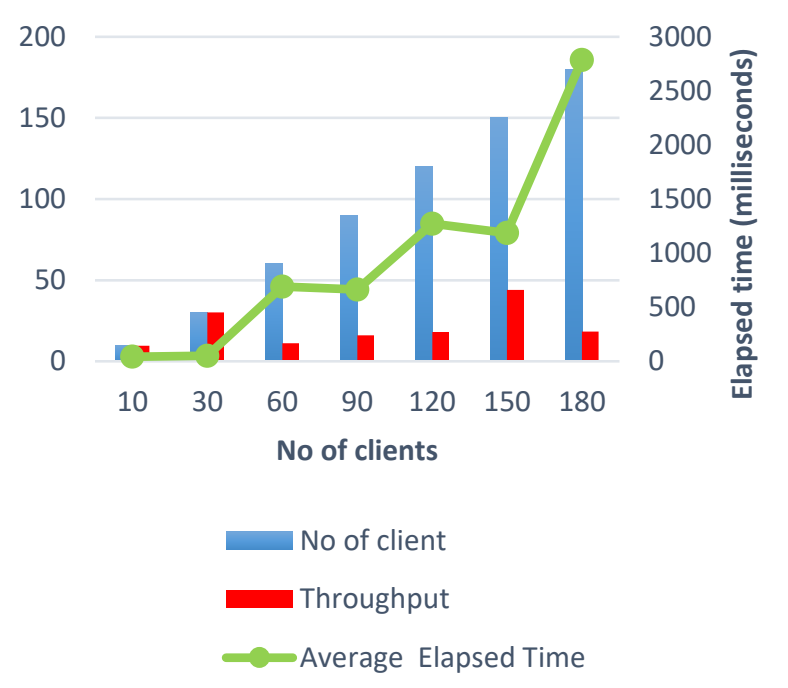

Figure 9. The average elapsed time and throughput relation with client no

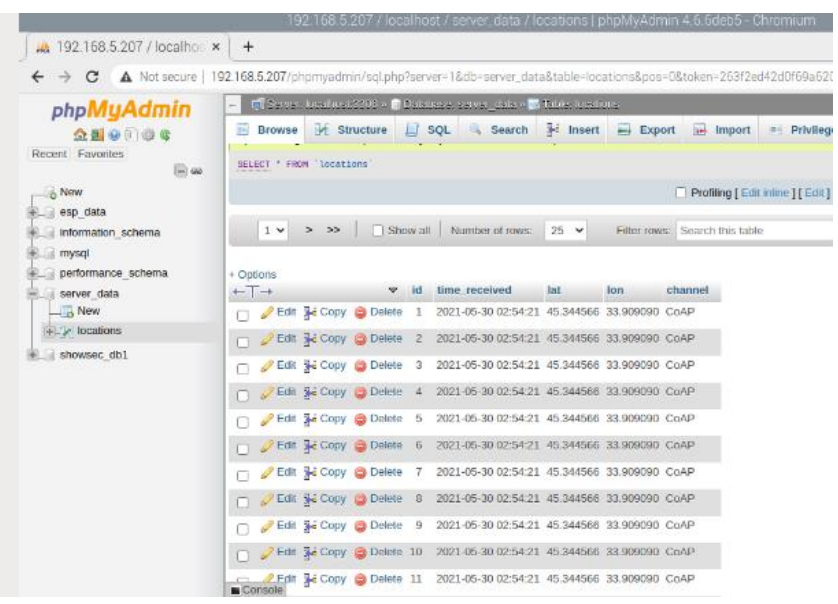

Figure 10. The data stored in MySQL

\section{Conclusion}

The proposed IoT cloud enables accurate realtime tracking at a low cost. These locations are collected and stored in the database at the same time of tracking. This approach can be used directly for many applications like urban planning of the smart city. Where the storage in the MySQL database familiar and easy to use for everyone. This study presents the IoT cloud-based on CoAP protocol where the Raspberry $\mathrm{Pi}$ acts as a cloud with MySQL storage services, and this cloud can connect with any end node like GPS sensors throw the internet. The study also tests the ability of the proposed architecture to handle with the maximum number of the client whom send the locations and store them in the one second. The Apache JMeter software (Windows system) is adopted to generate the virtual clients and at the same time measure standard division, average elapsed time, error rate, throughput for each load. From these results, we found that when the number of clients $(60,90$, and 120) there is some error rate because the clients don't receive a response (ACK) from a server after sending all requests. Still, all the data are stored in MySQL without any losses, and this time delay decreases the value of throughput as shown in Fig. 9. Because the throughput depends on successful requests handled by the server per unit of time. In the case of 180 clients, not all the data stored in MySQL. From these results, we found that the system could store 150 GPS locations per second without any loss.

\section{Acknowledgments}

The authors are grateful and would like to thank Al Mustansiriyah University (www.uomustansiriyah.edu.iq) for assisting in completing this article. Special thanks to the 
reviewers who are anonymous for their helpful feedback and positive comments.

\section{Conflict of interest}

The authors confirm that the publication of this article causes no conflict of interest.

$\begin{array}{ll}\text { Abbreviations } \\ \text { IoT } & \text { Internet of Things } \\ \text { CoAP } & \text { Constrained Application Protocol } \\ \text { GPS } & \text { Global Positioning System } \\ \text { RFID } & \text { Radio-frequency Identification } \\ \text { NIST } & \text { National Institute of Standers And } \\ & \text { Technology } \\ \text { HTTP } & \text { Hypertext Transfer Protocol } \\ \text { MQTT } & \text { Message Queuing Telemetry } \\ & \text { Transport } \\ \text { IETF } & \text { Internet Engineering Task Force } \\ \text { UDP } & \text { User Datagram Protocol } \\ \text { TCP } & \text { Transmission Control Protocol } \\ \text { VNC } & \text { Virtual Network Computing } \\ \text { URL } & \text { Uniform Resource Locator } \\ \text { IP } & \text { Internet Protocol }\end{array}$

\section{References}

1. Hajji, W., \& Tso, F. P. (2016). Understanding the performance of low power raspberry pi cloud for big data. Electronics (Switzerland), 5(2), 1-14. https://doi.org/10.3390/electronics502002 9

2. Sobin, C. C. (2020). A Survey on Architecture, Protocols and Challenges in IoT. In Wireless Personal Communications (Vol. 112, Issue 3). Springer US. https://doi.org/10.1007/s11277-02007108-5

3. Kristiani, E., Yang, C. T., Wang, Y. T., \& Huang, C. Y. (2011). The NIST Definition of Cloud Computing. Lecture Notes in
Electrical Engineering, 514, 675-685. https://doi.org/10.1007/978-981-13-10560_66

4. Babu, S. M., Lakshmi, A. J., \& Rao, B. T. (2015). A Study on Cloud based Internet of Things: CloudIoT. https://doi.org/10.1021/bi00565a004

5. Bastos, D. (2019). Cloud for IoT - A survey of technologies and security features of public cloud IoT solutions. IET Conference Publications, 2019(CP756). https://doi.org/10.1049/cp.2019.0168

6. Christos Stergiou a, Kostas E. Psannis a,*, Byung-Gyu Kimb, B. G. c. (2018). Secure integration of IoT and Cloud Computing.

7. Hou, L., Zhao, S., Xiong, X., Zheng, K., \& Member, S. (2016). Internet of Things Cloud: Architecture and Implementation. IEEE Communications Magazine, 1-19.

8. Scott, T. L., \& Eleyan, A. (2019). CoAP based IoT data transfer from a Raspberry $P i$.

9. Ugrenovic, D., \& Gardasevic, G. (2016). CoAP protocol for Web-based monitoring in IoT healthcare applications. 2015 23rd Telecommunications Forum, TELFOR 2015, 79-82. https://doi.org/10.1109/TELFOR.2015.737 7418

10. Daud, M. A., \& Suhaili, W. S. H. (2017). Internet of things (IoT) with coap and HTTP protocol: A study on which protocol suits IoT in terms of performance. Advances in Intelligent Systems and Computing, 532, 165-174. https://doi.org/10.1007/978-3-319-48517$1 \_15$

11. Kassab, W., \& Darabkh, K. A. (2020). A$\mathrm{Z}$ survey of Internet of Things: Architectures, protocols, applications, recent advances, future directions and recommendations. Journal of Network and Computer Applications, 163(April). https://doi.org/10.1016/j.jnca.2020.102663

12. Mughal, R. A. (2018). Internet of Things ( IoT ) Protocols: A Brief Exploration of MQTT and CoAP. May. https://doi.org/10.5120/ijca2018916438 
13. Anusha, M., Suresh Babu, E., Sai Mahesh Reddy, L., Vamsi Krishna, A., \& Bhagyasree, B. (2017). Performance analysis of data protocols of internet of things: A qualitative review. International Journal of Pure and Applied Mathematics, 115(6 Special Issue), 37-47.

14. Martí, M., Garcia-Rubio, C., \& Campo, C. (2019). Performance Evaluation of CoAP and MQTT_SN in an IoT Environment. Proceedings, 31(1), 49. https://doi.org/10.3390/proceedings 20190 31049

15. Araujo Soto, V. (2017). Performance evaluation of scalable and distributed IoT platforms for Smart Regions. 103.

16. starrlingo. (2017). jmeter-iot-lib_ $A$ storage to place current Jmeter plugin which support stress test on MQTT_MQTTS and COAP protocol. https://github.com/starrlingo/jmeter-iot-lib

17. RaspberryPi. (2016). Raspberry Pi 3 Model B+ Datasheet. In Datasheet (p. 5). https://static.raspberrypi.org/files/productbriefs/Raspberry-Pi-Model-Bplus-ProductBrief.pdf

18. Heba Mohammed Fadhil, Haneen Adel Mohammed, and M. S. J. (2019). Private Cloud Data Storage Using Raspberry Pi $\uparrow$. ICSES Interdisciplinary Transactions on Cloud Computing, 3(3), 1-5.

19. G. Tanganelli, C. Vallati, E. M. (2015). CoAPthon: Easy Development of CoAPbased IoT Applications with Python.

20. Shelby, Z., Hartke, K., \& Bormann, C. (2014). The Constrained Application Protocol (CoAP). In $R f c 7252$ (p. 112). https://www.rfceditor.org/rfc/pdfrfc/rfc7252.txt.pdf 